\title{
Metabolomics and its Applications in Cancer Cachexia
}

\author{
Pengfei Cui ${ }^{1}$, Xiaoyi $L^{2}{ }^{2}$, Caihua Huang ${ }^{3}$, Qinxi $L^{4}{ }^{4}$ and Donghai Lin ${ }^{5 *}$ \\ ${ }^{1}$ College of Food and Pharmacy, Xuchang University, Xuchang, China, ${ }^{2}$ Xuchang Central Hospital, Xuchang, China, ${ }^{3}$ Department \\ of Physical Education, Xiamen University of Technology, Xiamen, China, ${ }^{4}$ State Key Laboratory of Cellular Stress Biology, School \\ of Life Sciences, Xiamen University, Xiamen, China, ${ }^{5}$ Key Laboratory for Chemical Biology of Fujian Province, MOE Key Laboratory \\ of Spectrochemical Analysis and Instrumentation, College of Chemistry and Chemical Engineering, Xiamen University, Xiamen, \\ China
}

Cancer cachexia (CC) is a complicated metabolic derangement and muscle wasting syndrome, affecting 50-80\% cancer patients. So far, molecular mechanisms underlying CC remain elusive. Metabolomics techniques have been used to study metabolic shifts including changes of metabolite concentrations and disturbed metabolic pathways in the progression of CC, and expand further fundamental understanding of muscle loss. In this article, we aim to review the research progress and applications of metabolomics on CC in the past decade, and provide a theoretical basis for the study of prediction, early diagnosis, and therapy of CC.

Keywords: cancer cachexia, metabolomics, metabolic alterations, progress, biomarker

\section{OPEN ACCESS}

Edited by:

Wolfram Weckwerth,

University of Vienna, Austria

Reviewed by:

Cheng Guo,

Zhejiang University, China Jian-Bo Wan,

University of Macau, China

${ }^{*}$ Correspondence: Donghai Lin dhlin@xmu.edu.cn

Specialty section: This article was submitted to Metabolomics,

a section of the journal

Frontiers in Molecular Biosciences

Received: 05 October 2021

Accepted: 17 January 2022

Published: 07 February 2022

Citation:

Cui P, Li X, Huang C, Li Q and Lin D (2022) Metabolomics and its Applications in Cancer Cachexia.

Front. Mol. Biosci. 9:789889. doi: $10.3389 /$ fmolb.2022.789889

\section{INTRODUCTION}

Cancer cachexia (CC) is a multifactorial syndrome, which is characterized by disturbed metabolism, declined body weight, depleted muscle mass, and reduced food intake (Evans et al., 2008; Fearon et al., 2011). Overall, CC affects approximately 50-80\% of cancer patients and leads to around $30 \%$ of mortality, with the highest incidence reported in gastrointestinal and pancreatic cancers (Loberg et al., 2007; Kumar et al., 2010). Lately, four stages of CC have been proposed to define the guidelines (Bozzetti and Mariani, 2009; Blum et al., 2010). Initially, CC begins in a pre-cachexia stage with unwitting body weight loss, along with a more severe and noninvertible fat tissues and skeletal muscles loss, followed by disturbances in metabolic pathway and immune system, ultimately resulting in death (Hamerman, 2002; Deans et al., 2009).

Declined body weight primarily arise from skeletal muscle loss, which is recognized as the major feature of CC. Muscle loss makes routine activities difficult and results in tiredness, in addition to the tremendous damage to quality of life and poor response to surgery or chemotherapy (Lok, 2015). Study showed that treatment on skeletal muscle loss could not only attenuate the symptoms of CC, but also remarkably prolongs lifespan (Zhou et al., 2010). Previous studies have found that CC is linked to various factors including fasting hormones, pro-inflammatory cytokines, such as interleukin 1 (IL-1), tumor necrosis factor-alpha (TNF- $\alpha$ ), interferon-gamma (IFN- $\gamma$ ) (Nagaya et al., 2006; Gupta et al., 2011; Argiles et al., 2013; Argiles and Stemmler, 2013). The two main cell proteolysis pathways including ubiquitin-proteasome pathway and autophagy pathway regulate protein turnover in muscle tissues (Bonaldo and Sandri, 2013; Halle et al., 2020; Lim et al., 2020; Yang et al., 2020). In addition, several major signaling pathways including IGF1-Akt-FoxO pathway, TGF $\beta$-myostatin pathway, NF- $\kappa \mathrm{B}$ signaling, and glucocorticoids pathway have all been implicated in muscle atrophy of CC (Bodine et al., 2001; Musaro et al., 2001; Lee, 2004; Sandri et al., 2004; Waddell et al., 2008; Peterson et al., 2011). Identification of signaling pathways associated with CC and muscle atrophy has achieved great progress in recent decades. Given that CC is a typical metabolic 
syndrome, metabolomic techniques can be applied to explore biomarkers for early diagnosis of CC, address metabolic characteristics for mechanistic understanding of the pathogenesis of CC, and develop therapeutics strategies for treatments of CC.

As an omics technology developing after genomics, transcriptomics and proteomics, metabolomics has rapid developments at present, which can simultaneously analyze all of metabolites with small molecular weights in a biological system (Newgard, 2017). Compared to genomics, transcriptomics and proteomics, metabolomics is based on extensively used detection equipment including either mass spectrometry (MS) or nuclear magnetic resonance spectroscopy (NMR), which has the features of high sensitivity, high precision, good resolution, and small sample volume (Beckonert et al., 2007; Ma et al., 2018). In the last 2 decades, metabolomic techniques have been extensively used to exploring various diseases such as cancer (Wishart, 2016; Schmidt et al., 2021), type 2 diabetes (Newgard et al., 2009; Ma et al., 2018), fatty liver (Gao et al., 2009; Lallukka and Yki-Jarvinen, 2016), and cardiovascular diseases (Shah et al., 2012a; Shah et al., 2012b).

Metabolomic techniques create ideas and clues for scholars to predict and screen CC at early stage. Recently, researchers have applied metabolomic analysis to perform global and in-depth studies for identifying metabolic signatures in patients or animal models or cell models with CC, and also for identifying potential biomarkers and crucial metabolic pathways to mechanistically understand the pathogenesis of CC. We searched for articles from PubMed, Scopus and Google Scholar relevant to cancer cachexia by using the keywords "cancer cachexia and metabolomics," "cancer cachexia and metabonomics," "cancer cachexia and metabolic," "muscle atrophy and metabolomics," "muscle loss and metabolomics" and so on. We have only included the studies based on the animal models or clinical samples related to CC by using metabolomics methodologies. So far, only two reviews of omics studies on CC have been reported (Gallagher et al., 2016; Twelkmeyer et al., 2017). However, these reviews did not pay much attention to the field of metabolomics. To widely expand the knowledge of CC and give inspirations for the cachexia studies from the view of biomarkers, signatures and therapeutic targets, we focus on the progress made in the past decade, novel developments, and latest discoveries in the study of CC using metabolomic techniques, and look forward to its future developments. To the best of our knowledge, this article presents the first review on the progress of metabolomic applications in CC.

\section{METABOLOMIC RESEARCH METHODOLOGIES AND TECHNIQUES}

Followed by genomics, transcriptomics, and proteomics, metabolomics is a promising subject that has promptly developed in recent years. It can be qualitatively and quantitatively employed to analyze various sample sources, which include cells or tissues extract, bio-fluids, and microorganisms caused by genetically engineered or drug treatment. Metabolomic analyses usually focus on small molecular metabolites such as amino acids, lipids, small molecular peptides, and organic acids with a relative molecular weight of less than 1,000 Da (Nicholson et al., 1999; Fiehn et al., 2000; Xia and Wan, 2021). Generally, metabolomics includes two tools: non-targeted and targeted metabolomics. Non-targeted metabolomics is most widely used in CC studies to explore biomarkers (Yang et al., 2018), signatures (Cui et al., 2019b), and therapeutic targets (Fukawa et al., 2016). The process of metabolomic analysis in CC is depicted in Figure 1, which contains sample sources, analytical platforms, data collection and analysis, biomarkers identification, metabolic pathways exploration, and biological significance elucidation.

\section{Sample Sources}

The most commonly used type of samples for metabolomic studies in CC are serum/plasma, urine, tumor tissues, liver and skeletal muscle, and other tissues. The collected blood samples are further processed with cell separation to obtain sera and plasma at $4^{\circ} \mathrm{C}$ before analysis. This step might be one of the major factors of pre-analysis errors in blood metabolomics research (Beckonert et al., 2007; Nikolic et al., 2014). It is generally suggested that the interval between blood sample collection and cell separation should be finished in $35 \mathrm{~min}$ to avoid the increased lactate levels. In addition, repeated freezing and thawing steps should be avoided in the whole experiment (Yin et al., 2015). Compared with blood samples, the biological composition of urine samples is relatively simple, the protein content is low, and additional metabolite extraction steps are not usually required. The commonly used pretreatment method for skeletal muscle and tumor tissues in CC studies is liquid-liquid extraction. In general, tissue samples are initially extracted with cold solutions which contains chloroform, methanol and water in a certain ratio to generate a two-phase system. The polar and nonpolar metabolites are separated, lyophilized and dissolved in corresponding solvents, respectively (Jonsson et al., 2005; Beckonert et al., 2007; Legido-Quigley et al., 2010).

\section{Data Collection Techniques}

Metabolomic techniques are applied to measure the number, type, condition, and level of metabolites and to explore metabolic profiles (Beckonert et al., 2007). Compared to other detection techniques, NMR and MS are the two mostly used techniques for metabolomic analysis (Emwas, 2015).

NMR technology is a spectroscopic technology that uses different atomic nuclei to absorb ratio-frequency radiation with different resonance frequencies, which are converted into molecular chemistry and structural information related to environments of the nuclei (Bothwell and Griffin, 2011). With the development of NMR technology, researchers can directly analyze intact gastrocnemius muscle without any pretreatment of samples by using high-resolution magic angle rotation (HRMASNMR) spectroscopy in a CC mouse model (Yang et al., 2015). Overall, NMR spectroscopy has many advantages such as simple sample preparation, non-invasive and unbiased measurement of the sample, good objectivity and reproducibility (Li et al., 2015). However, signal overlap and low sensitivity are two obvious shortcomings in complicated ${ }^{1} \mathrm{H}$-NMR spectra. 


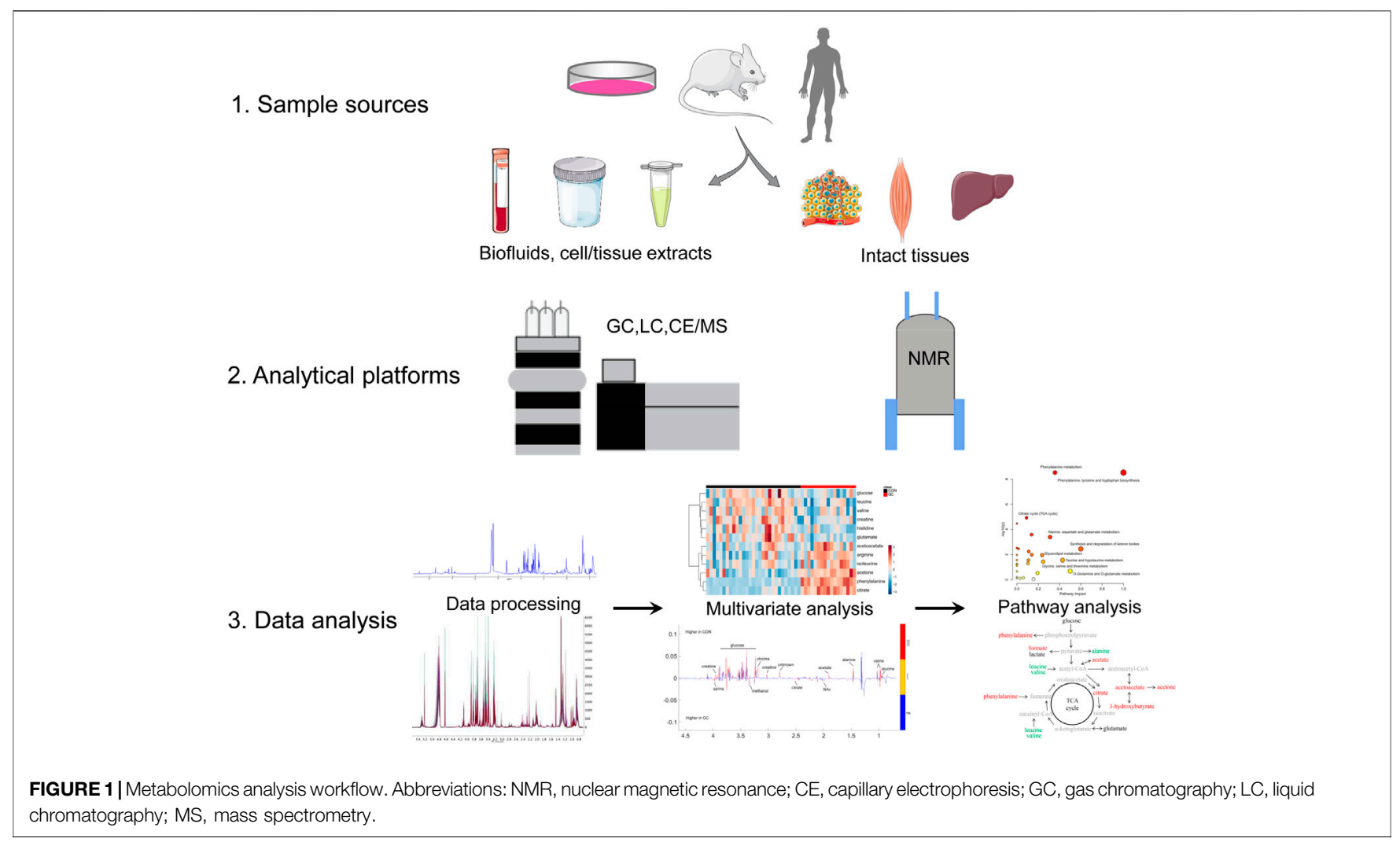

TABLE 1 | Summarization of advantages and drawbacks of MS and NMR detections.

Features

Sample preparation

Sample measurement

Sample recovery

Selectivity and targeted analysis capabilities

Sensibility

Resolution

Repeatability
NMR

Simple
Simple
Good, non-invasive
General, mostly in untargeted analysis
Low, <100 metabolites per test
General
High

MS

Complex

Complex, various chromatography methods Destructive

Good, untargeted and targeted analysis High, >1,000 metabolites per test

General

low
MS spectroscopy uses electric and magnetic fields to separate moving ions and detect them according to the mass-to-charge ratio (m/z) (Tsiropoulou et al., 2017). At present, MS combined with chromatography are divided into three types including capillary electrophoresis-mass (CE-MS), gas chromatographymass (GC-MS), and liquid chromatography-mass (LC-MS). CE-MS has high performance for polar and ionic compounds with high resolution and sensitivity rather than uncharged compounds (Ramautar et al., 2019; Stolz et al., 2019). Compared to GC and LC, CE has a superiority over them for the resolution of charged molecules along with the isomers due to the excellent separation. GC-MS is generally conducted to analyze non-polar, low-boiling and volatile molecules, and samples usually need to be derivatized. LC-MS has relatively high sensitivity and strong detection ability for polar and thermally unstable compounds, by which a wider range of metabolites with low detection limit can be analyzed. It can be used for trace analysis and is more suitable for metabolomic analysis of complex biological samples (Römisch-Margl et al., 2011; Xiao et al., 2012). Cala and colleagues performed a combination of 3 types of MS (GC-MS, CE-MS, and LC-MS) to obtain plasma metabolite fingerprinting in a CC clinical study (Cala et al., 2018).

Compared with NMR spectroscopy, MS has several advantages such as high sensitivity and resolution, which could detect thousands of metabolites in a large dynamic range at the same time. However, MS also has its own shortcomings such as complicated sample preparation and low reproducibility. The advantages and drawbacks of MS and NMR detections are listed in Table 1 . To promote the entire performance of metabolomics studies, Pin and colleagues combined MS and NMR to investigate differences between CC and chemotherapy induced cachexia (Pin et al., 2019).

\section{Data Preprocessing and Analysis}

Data analysis includes data preprocessing, multivariate statistical analysis, model establishment and verification, and selection of 
difference variables, etc. Prior to obtaining metabolomics data for statistical analysis, it is necessary to preprocess the data, which mainly includes baseline correction, peak screening (peak identification, peak alignment and correction), noise filtering, missing value processing, normalization and scaling (Dunn et al., 2011). Thereafter, multivariate statistical analysis is conducted to decrease the dimensionality of acquired data and extract information, including principal component analysis (PCA), clustering analysis, partial least square analysis (PLS), PLSdiscriminant analysis (PLS-DA), orthogonal PLS (OPLS)-DA and random forests (RF) (Idborg-Bjorkman et al., 2003; Wiklund et al., 2008; Sugimoto et al., 2012; Schwammle et al., 2015). PCA, PLS-DA, OPLS loading plot and heatmap analysis were most commonly used in CC metabolomics studies. Besides, general statistical analyses including analysis of variance (ANOVA) and Student's t-test are also applied to quantitatively analyze the abundance of metabolites between different groups. When performing the multiple comparisons, the familywise error rate (FWER) might cause false-positive detection, which could be diminished by the procedures of false discovery rate (FDR) with Holm, Bonferroni and Benjamini-Hochberg corrections in metabolomic analysis (Sugimoto et al., 2012; Muroya et al., 2020). Overall, the combination of multivariate statistical analysis and classical statistical analysis can improve the reliability of the data analysis.

\section{Data Elucidation}

After multivariate statistical analysis, one can uncover and illustrate metabolic signatures based on several databases, including significantly altered concentrations of metabolites and certain disturbed metabolic pathways corresponding to external metabolic stimuli. These databases include HMDB (http://www.hmdb.ca/), METLIN (https://metlin.scripps.edu/), SMPDB (https://smpdb.ca), MassBank (http://www.massbank. $\mathrm{jp} /$ ), The Kyoto Encyclopedia of Genes and Genomes (KEGG; https://www.genome.jp/kegg/), and software such as MetaboAnalyst 5.0 (https://www.metaboanalyst.ca/) (Pang et al., 2021). A growing number of studies have been using MetaboAnalyst website to conduct the pathway analysis and ROC analysis in CC studies (Yang et al., 2018; Cui et al., 2019b; Sadek et al., 2021).

\section{ADVANCES IN THE PATHOGENESIS OF CANCER CACHEXIA BASED ON METABOLOMICS}

Skeletal muscle loss might occur in the early stage, which might be masked by dysfunction and symptoms of other tissues. Methods used to assess muscle loss involve diagnostic imaging techniques, including computed tomography (CT), dual energy X-ray absorptiometry (DXA), and magnetic resonance imaging (MRI). However, these methods are associated with several shortcomings such as time consuming, expensive, complicated, and invasive when clinicians wish to screen the early or slow muscle loss (Heymsfield et al., 1997; Mitsiopoulos et al., 1998; Shen et al., 2004; Mourtzakis et al., 2008). In order to exploit the progress of muscle loss dynamically, several methods have been developed to detect CC syndromes and shorten the period for early prevention (Evans et al., 2008). Recently, metabolomic analysis is widely being applied to uncover novel biomarkers, explore certain metabolic pathways associated with the pathogenesis of various diseases including CC, and ultimately exploit potential therapeutic strategies in the future (Twelkmeyer et al., 2017). Applications of metabolomics analysis in CC are depicted in Figure 2, which cover biomarkers, signatures and therapeutic targets.

\section{Biomarkers}

Metabolomics can be applied to detect hundreds of small metabolites simultaneously for providing better elucidation of metabolic pathways related to the pathological mechanisms of $\mathrm{CC}$, ultimately identifying reliable biomarkers for diagnosis and monitoring of cachexia.

Metabolomics studies in CC began in 2008 relied on the classical colon-26 (C26) mouse model. Connell and colleagues demonstrated that metabolomic analysis has the ability to diagnose and discover the surrogate serum biomarkers in CC for the first time (O'Connell et al., 2008). They conducted NMRbased metabolomic analysis on serum samples, and observed significant metabolic alterations including elevated amounts of very low-density lipoprotein (VLDL) and low-density lipoprotein (LDL) related to aberrant glycosylation of $\beta$-Dystroglycan (O'Connell et al., 2008). In a recent study based on the same C26 model, Lautaoja and colleagues identified free phenylalanine in sera and muscle tissues as a promising biomarker of cachectic muscle atrophy by using GC-MS-based metabolomic analysis (Lautaoja et al., 2019).

Furthermore, Kunz and colleagues performed untargeted LCMS-based metabolomic analysis of plasma and skeletal muscle in a Lewis lung carcinoma (LLC) mouse model. They detected increased levels of asymmetric dimethylarginine, and NGmonomethyl-L-arginine in LLC group relative to normal group. In order to further explore the function of these two methylarginines in muscle turnover, the researchers treated the cultured myotubes with these two metabolites and found impaired muscle protein synthesis in vitro study. Surprisingly, increased levels of asymmetric dimethylarginine were also observed in muscle tissues from clinic patients. This study not only discovered two novel potential biomarkers, but also provided therapeutic ideas for CC (Kunz et al., 2020).

In addition, Yang and colleagues revealed dynamically changing metabolic profiles in sera and intact muscle of CC in the C26 mouse model from pre-cachexia to the refractory cachexia period. They identified five unique metabolic features including declined levels of serum glucose and BCAAs, increased levels of ketone bodies, neutral amino acids and 3methylhistidine (Yang et al., 2015). Using HRMAS-NMR spectroscopy, they performed metabolic profiling of cachectic gastrocnemius muscle for the first time. To further validate the metabolic features identified from the mouse model, recently, Yang and colleagues recruited 33 pre-cachectic, 84 cachectic and 105 cancer patients with stable body weights and 74 healthy controls, according to the international definition and 


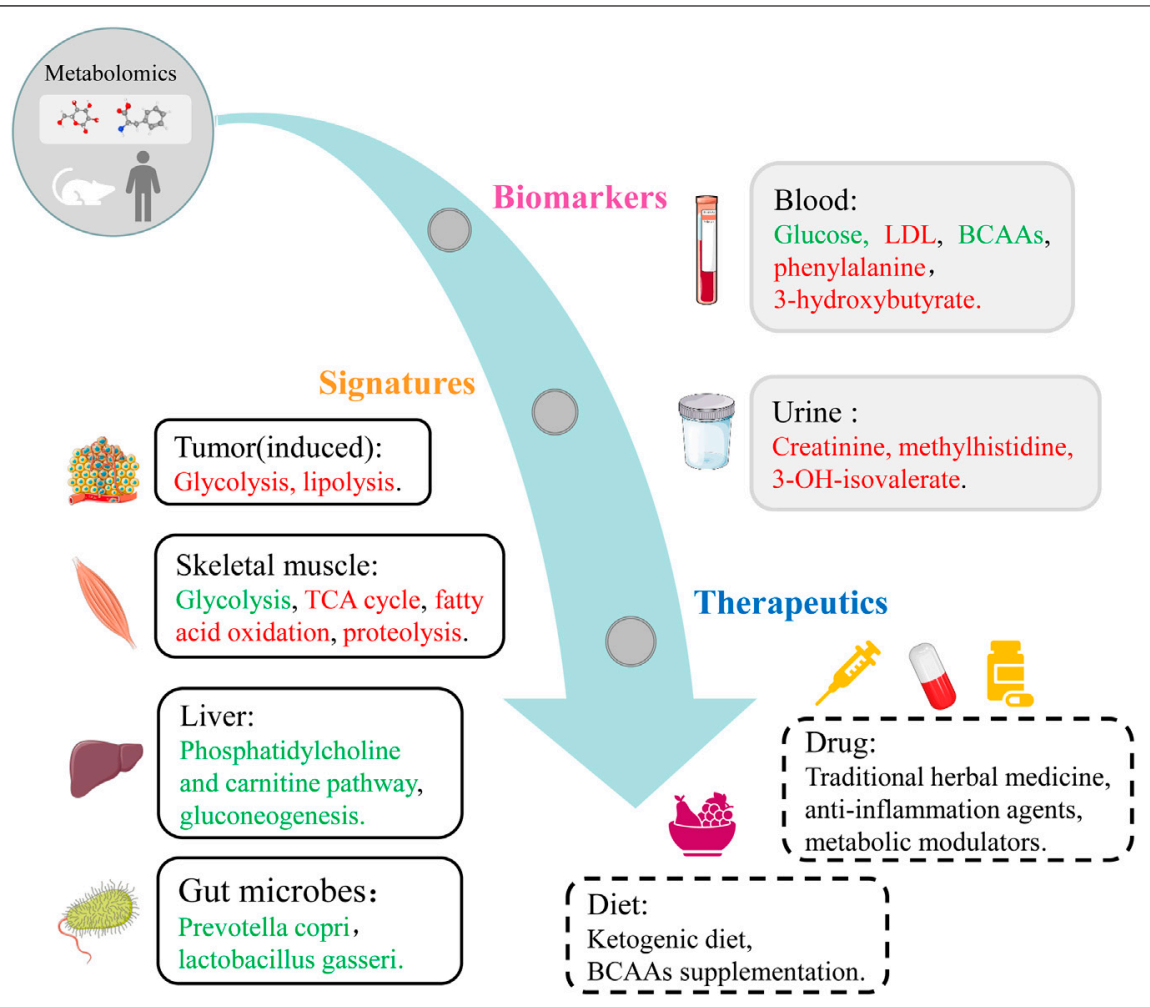

FIGURE 2 | Metabolomics applications covering biomarkers, signatures and therapeutic targets in CC. Red, upregulated metabolites and pathways in CC group. Green, downregulated metabolites, microbes and pathways in CC group.

classification of CC (Fearon et al., 2011). They conducted NMR metabolomic analyses on sera and urine of CC patients to reveal the metabolic profile of $\mathrm{CC}$, and identified 15 metabolites for discriminating different disease states (Yang et al., 2018). Based on three identified metabolites (carnosine, leucine and phenyl acetate), they established a diagnostic model for predicting the presence of cachexia with high accuracy.

In a previous study, Fujiwara and colleagues enrolled 21 advanced pancreatic cancer patients with or without cachexia, collected serum samples at different time point, and performed GC-MS-based metabolomic analysis (Fujiwara et al., 2014). They observed intraday differences in serum metabolite concentration, which were observably altered in the evening but basically identical in the daytime. Specifically, abundance of paraxanthine was significantly decreased in CC patients compared to those without cachexia all day long, which was potentially associated with cachexia. Additionally, another study performed NMR-based metabolomics analysis on 170 patients with head and neck squamous cell carcinoma cancer (HNSCC). These patients experienced radical treatments with radio-/chemo-radiotherapy (RT/CHRT) (Boguszewicz et al., 2019). Boguszewicz and colleagues indicated that serum metabolic alterations primarily related to high 3-hydroxybutyrate levels could be detected at an early stage of the treatment experienced by HNSCC patients. Thus, 3-hydroxybutyrate could be exploited as a fast and sensitive biomarker of malnutrition or cachexia.

Similarly, Miller and colleagues conducted LC-MS-based metabolomic analysis and identified potential biomarkers related to weight loss in patients with upper gastrointestinal cancer, which could be applied for the assessment of therapeutic intervention (Miller et al., 2019). Cancer patients with $\geq 5 \%$ weight loss displayed plasma metabolic profiles distinguished from those with $<5 \%$ weight loss. Totally, six metabolites were highly discriminative of body weight loss, including lysoPC18.2 and 16:1, hexadecanoic acid, octadecanoic acid, phenylalanine.

Metabolites in urine samples have also been investigated to discover novel biomarkers for CC. Eisner and colleagues did the first attempt to use single time-point urinary metabolite profiles to diagnose muscle wasting occurring in CC humans (Eisner et al., 2011). After analyzing 93 random urine samples from cancer patients, the researchers found that some metabolites such as creatinine and methylhistidine arising from muscle proteolysis were particularly released into urine. This study provides an inspiration that it might also be convenient, cheap and safe to detect muscle wasting based on ${ }^{1} \mathrm{H}-\mathrm{NMR}$ urine metabolomic analysis. Overall, these results obtained from previous studies on biomarkers for CC mostly depend on the samples derived from animal models and human, and also on tumor type, bio-fluids and analytical platforms.

\section{Metabolic Signatures and Metabolic Pathways}

Although numerous researches have been exploring molecular mechanisms underlying muscle wasting in CC, the effect of 
muscle wasting on muscle function and metabolic signatures remains unclear (Diffee et al., 2002). Metabolic impairments in the skeletal muscle are related to its physiological dysfunction. Thus, metabolic derangements might be involved in molecular mechanisms underlying protein synthesis and breakdown (Tisdale, 2003; Santarpia et al., 2011).

Yang and colleagues indicated that serum metabolic disturbances associated with promoted tricarboxylic acid (TCA) cycle and amino acid metabolism were the major features of CC in C26 mouse model. Amino acids, ketone bodies and metabolites involved in TCA cycle were recognized as potential biomarkers related to the corresponding metabolic pathways (Quanjun et al., 2013). Furthermore, Torossian and colleagues performed GC-MS-based and LC-MS-based metabolomic analyses to reveal metabolic distinctions between cachectic gastrocnemius muscles and control muscles in the C26 mouse model (Der-Torossian et al., 2013b). They showed predominant effects of CC including: enhanced oxidative stress, impaired redox homeostasis, altered metabolite concentrations in glycolysis and declined carbon flow through TCA cycle. This study found the tumor Warburg-like metabolic pattern in skeletal muscle of CC for the first time, which is considered as novel metabolic signature in CC research.

Compared to malabsorption, fasting, age-induced muscle loss, and sarcopenia, CC has its own metabolic features (Fearon, 1992; Tisdale, 2009; Evans, 2010; Rolland et al., 2011). Consistently, Torossian and colleagues performed a NMR-based metabolomic analysis of sera, and indicated that metabolic alterations including hyperlipidemia, hyperglycemia and reduced BCAAs distinguish cachexia from effects of starvation (Der-Torossian et al., 2013a). Another previous study explored metabolic differences between sarcopenia and CC in senile cancer animals. The researchers conducted NMR-based metabolomic analysis dynamically on sera derived from adult and ageing rats. The metabolic alterations mostly focused in several metabolic pathways, including amino acid biosynthesis which was upregulated in the aging group and downregulated in the tumor groups (Viana et al., 2020). Recently, they also performed NMR-based metabolomic analysis on gastrocnemius derived from weanling and young adult rats, aiming to explore metabolic alterations in cachectic hosts during the whole lifespan (Chiocchetti et al., 2021). They indicated that the most significant variations of metabolites such as glutamate, glutamine, glycine, and methylhistidine, might be associated with the early muscle catabolism and declined energy generation in cachectic muscles.

Chemotherapy is widely used to cancer patients in the clinical treatment, however, growing evidences have shown that several chemotherapeutic drugs could also lead to the occurrence of cachexia and deterioration of muscle mass. To date, only one metabolomics investigation has been done in chemotherapyinduced cachexia. Based on the C26 mouse model, Pin and colleagues revealed significant differences in amino acid catabolism, TCA cycle, and $\beta$-oxidation between CC and chemotherapy-induced cachexia by a combination of NMRbased metabolomics with targeted MS analysis (Pin et al., 2019).

Although skeletal muscles are the main tissue impaired dramatically in CC, other tissues such as liver and gut may also be affected and involved in the pathophysiology of this complex syndrome (Rohm et al., 2019). As an essential metabolic organ, liver regulates body energy metabolism and maintains its homeostasis. Dysfunction of liver metabolism are prone to cause promoted energy consumption in CC. Furthermore, gut microbial species play key roles in nutrients supplementation, cytokines and gut hormones regulation, and gut barrier function improvement. Based on these beneficial effects, scholars are exploring if these micrograms could act as novel therapeutic targets for CC (Valdes et al., 2018). Based on the C26 mouse model, Pötgens and colleagues explored the crosstalk in four different samples including caecal, portal vein, vena cava and liver by a combination of NMR-based metabolomics with gut gene sequencing and hepatic transcriptomics. Their results showed depressed glycolysis and gluconeogenesis, activated hexosamine pathway and phosphatidylcholine pathway, reduced abundances of hepatic carnitine and caecal acetate and butyrate, and decreased levels of aromatic amino acids (Potgens et al., 2021). Given that CC also induces anorexia and reduced food intake, Uzu and colleagues focused on studying metabolic signatures of brains and conducted a CEMS-based metabolomic analysis on brain samples derived from a CC mouse model. They observed activated purine metabolism and increased xanthine oxidase activity in brains of cachexic mice relative to controls (Uzu et al., 2019).

$\mathrm{Ni}$ and colleagues conducted a comprehensive analysis on 31 patients with lung cancer by a combination of plasma metabolomics and gut microbiota metagenomics (Ni et al., 2021). For the first time, they explored gut microbiota functions in the clinical CC study, and observed remarkably decreased levels of BCAAs, methylhistamine, and vitamins in CC blood. They further discovered that increased levels of BCAAs and 3-oxocholic acid in non-CC blood were closely related to gut microbiota especially Prevotella copri and Lactobacillus gasseri, respectively. These results shed lights on molecular mechanisms underlying host-microbiota crosstalk in CC, and provided new strategies for preventing or treating CC through regulating gut microbiota in the future nutritional supplements.

Previously, preclinical mouse models (mainly C26 and LLC) were established by using subcutaneous implantation methods to conduct CC studies. Few murine models of CC with orthotopic implantation have been employed. Thus, our group established two orthotopic models including glioma cachexia and gastric cancer cachexia to mimic clinical characteristics of CC. In the first study, we conducted NMR-based metabolomic analysis to explore metabolic profiles in cachectic muscle based on a glioma induced cachexia murine model (Cui et al., 2019b). Our results indicated that significantly impaired pathways including energy metabolism, muscle protein breakdown and synthesis, and profoundly increased amino acids involved in TCA cycle anaplerotic. After that, we established a gastric CC murine model and performed NMR-based metabolomic analysis of gastric tissues (tumor), blood and skeletal muscle. Cachectic mice exhibited impaired glucose and nucleic acid metabolisms in tumor, hyperlipidemia and hypoglycemia in blood, and disturbed carbohydrate and amino acid metabolism in gastrocnemius (Cui et al., 2019a). Besides, we further explored 
the role of a-ketoglutarate in muscle protein turnover, and found a-ketoglutarate can alleviate the myotubes atrophy induced by glucose deprivation.

At present, only one study was performed using a combination of three metabolomics techniques (GC-MS, CE-MS, and LC-MS) to access a markedly different metabolic pattern in human plasma (Cala et al., 2018). Cala and colleagues collected two groups of plasma samples from 8 cachectic and 7 non-cachectic patients (Cala et al., 2018). Their results exhibited significantly decreased levels of amino acids and glycerophospholipids, and increased cortisol levels associated with cachexia. The disturbed metabolic pathways in CC included amino acid metabolism, aminoacyltRNA biosynthesis, fatty acid elongation, and TCA cycle. In another study, Stretch and colleagues investigated metabolic profiles of urine and plasma derived from 55 weight-losing patients by conducting NMR-based and direct injection MSbased metabolomics analyses. Their results indicated that large amounts of glycerophospholipids variations can be used to discover sarcopenia in cancer patients (Stretch et al., 2012). This study addressed one main issue that the variability of tissue mass might impact metabolic profiles, and thus could provide hints for the field of nutrition and metabolism studies. Overall, numerous researchers have investigated the metabolic signatures for CC from various aspects such as starvation, sarcopenia, chemotherapy, gut microbes, orthotopic implantation and analytical platforms, in order to give clues and inspirations to better elucidate the pathogenesis of CC and therapeutic targets discovery.

\section{Therapeutic Strategies by Using Metabolomics}

As discussed above, metabolomics is being extensively used to uncover biomarkers, metabolic signatures and metabolic pathways of CC, and to exploit novel drug targets. In this section, we discuss studies on how metabolomics contributes to the discovery of new targets for therapy.

Gut microbiota could depress inflammation response and tumor development (Bindels et al., 2012). Some researchers have been exploring the roles of gut microbiome in CC and addressing certain metabolic signatures in the last section. Bindels and colleagues performed a further study on gut microbiota with the expectation of finding novel interventions for CC treatments. They integrated gene sequencing and metabolomics as well as molecular profiling of the host, so as to obtain a comprehensive view on the pathophysiology of CC (Bindels et al., 2016). The portal metabolome reflected significantly decreased glucose and lipoproteins levels, increased creatine and lactate levels. These data demonstrated that gut microbiota can impact intestinal homeostasis, confer benefits to the host, prolong survival and attenuate cachexia.

Increased expressions of inducible nitric oxide synthase (iNOS) have been observed in muscle tissues of cancer, AIDS, chronic heart failure, and COPD cachexia patients, suggesting that iNOS may be involved in the onset of cachexia under various conditions (Adams et al., 2003; Agusti et al., 2004; Ramamoorthy et al., 2009). Sadek and colleagues identified a signature of amino acids that were altered by iNOS activity in muscle by performing LC-MS-based and GC-MS-based metabolomic analyses based on the C26 murine model (Sadek et al., 2021). Notably, iNOS could significantly increase levels of arginine, lysine, tryptophan and methylhistidine, which could be decreased by inhibiting iNOS. Furthermore, they also found iNOS-induced significant decreases in levels of pyruvate, a-ketoglutarate and succinate, which were restored by $\mathrm{KO}$ iNOS. These results demonstrated that drug blockade or gene knockout of iNOS could rescue muscle loss and improve metabolic disorders in CC. This study provided the idea on how to use metabolomic techniques to identify potential targeted metabolic pathways. Initially, the researchers clarified metabolic alterations in animal models by conducting metabolomics analysis, thereafter they conducted genetic or pharmacological inhibition of iNOS on certain metabolic pathways including glycolysis, TCA cycle and fatty acid oxidation, which were all related to the energy production. Ultimately, they clearly elucidated the role of the iNOS/NO pathway in promoting energy crisis during cachexia-induced muscle wasting.

In addition, Ballarò and colleagues found that abnormal muscle mitochondrial function is correlated with excessive proteolysis, autophagy and mitophagy in the established CC model (Penna et al., 2019). They conducted NMR-based metabolomic analyses of skeletal muscle, liver and plasma. They identified significantly altered energy and protein metabolism such as decreased muscle NADH, increased glutamine, BCAAs and phenylalanine in tumor hosts. Partially, mitochondria-targeted compound SS-31 could modulate both skeletal muscle metabolome and liver metabolome, restore levels of alanine and ATP, as well as liver glycogen and glutathione. This study suggested that targeting mitochondrial function might be an efficient therapeutic approach for CC (Ballaro et al., 2021).

Researches have illustrated that intervening targeted metabolic pathways could attenuate CC symptoms and prevent muscle loss. Yang and colleagues investigated metabolic signatures of CC and the contribution of formoterol to serum metabolites in the C26 mouse model with NMR-based metabolomics approach. They identified several potential biomarkers including amino acids, ketone bodies and citrate cycle metabolites, which well reflected the effects of formoterol treatment (Quanjun et al., 2013). In a later study, this group conducted NMR-based metabolomic analysis based on the C26 mouse model. They exhibited that primary disturbed metabolic pathways in CC were biosynthesis of the BCAAs and glycine, serine, and threonine metabolism. Significantly, treatment with curcumin changed glycolysis with declined levels of lactate, alanine and glucose (Quan-Jun et al., 2015). In addition, Ohbuchi and colleagues exploited molecular mechanisms under the effects of rikkunshito (RKT) acting as a Japanese traditional herbal medicine (Kampo) for the treatment of CC. The researchers performed GC-MS-based plasma metabolomic analysis based on a rat model, and indicated that increased plasma glucarate following the RKT administration could delay body weight loss, reduce muscle wasting and ascites content (Ohbuchi et al., 2015). These studies shed lights on applications of traditional medicines for alleviating the progression of CC. 
TABLE 2 | Overview of metabolic characteristics of CC.

\begin{tabular}{|c|c|c|c|c|}
\hline References & $\begin{array}{l}\text { Study } \\
\text { object }\end{array}$ & $\begin{array}{l}\text { Sample } \\
\text { information }\end{array}$ & $\begin{array}{l}\text { Analytical } \\
\text { technology }\end{array}$ & Metabolic characteristics \\
\hline O'Connell et al. (2008) & Mice & Serum & NMR & $\begin{array}{l}\text { UP: VLDL/LDL; } \\
\text { DOWN: glucose. }\end{array}$ \\
\hline Eisner et al. (2011) & Patients & Urine & NMR & $\begin{array}{l}\text { UP: creatine, creatinine, } \\
\text { 3-OH-isovalerate. }\end{array}$ \\
\hline Stretch et al. (2012) & Patients & $\begin{array}{l}\text { Urine, } \\
\text { Plasma }\end{array}$ & $\begin{array}{l}\text { NMR, } \\
\text { MS }\end{array}$ & Glycerophospholipids and metabolites associated with amino acid metabolism. \\
\hline Quanjun et al. (2013) & Mice & Serum & NMR & Enhanced citrate cycle and amino acid metabolism. \\
\hline $\begin{array}{l}\text { Der-Torossian et al. } \\
\text { (2013a) }\end{array}$ & Mice & Serum & NMR & $\begin{array}{l}\text { Hyperlipidemia, hyperglycemia; } \\
\text { DOWN: BCAAs. }\end{array}$ \\
\hline $\begin{array}{l}\text { Der-Torossian et al. } \\
\text { (2013b) }\end{array}$ & Mice & Muscle & LC-MS & $\begin{array}{l}\text { Enhanced Warburg effect; } \\
\text { Disrupted TCA cycle, promoted oxidative stress, } \\
\text { impaired redox homeostasis. }\end{array}$ \\
\hline Fujiwara et al. (2014) & Patients & Serum & GC-MS & Down: paraxanthine. \\
\hline Ohbuchi et al. (2015) & Rat & Plasma & GC-MS & DOWN: glucarate; \\
\hline Yang et al. (2015) & Mice & Serum, Muscle & NMR & $\begin{array}{l}\text { UP: neutral amino acids, creatine, ketone bodies, 3-methylhistidine; } \\
\text { DOWN: BCAAs, glucose. }\end{array}$ \\
\hline Quan-Jun et al. (2015) & Mice & Serum & NMR & $\begin{array}{l}\text { UP: phenylalanine; } \\
\text { DOWN: BCAA, acetoacetate. }\end{array}$ \\
\hline Tseng et al. (2015) & Mice & Muscle & LC-MS & Impaired glycolysis, glycogen synthesis; protein degradation. \\
\hline Viana et al. (2016) & Rat & $\begin{array}{l}\text { Serum, } \\
\text { Tumor }\end{array}$ & NMR & UP: tryptophan, lactate, ketone bodies. \\
\hline Bindels et al. (2016) & Mice & Portal plasma & NMR & $\begin{array}{l}\text { UP: creatine, lactate; } \\
\text { DOWN: glucose, lipoproteins. }\end{array}$ \\
\hline Fukawa et al. (2016) & Mice & Muscle, cell & LC-MS & Excessive fatty acid oxidation, enhanced oxidative stress. \\
\hline Yang et al. (2018) & Patients & Serum, urine & NMR & $\begin{array}{l}\text { UP: Carnosine, phenylacetate; } \\
\text { Down: leucine. }\end{array}$ \\
\hline Cala et al. (2018) & Patients & Plasma & $\begin{array}{l}\text { LC-MS, } \\
\text { GC-MS, } \\
\text { CE-MS }\end{array}$ & $\begin{array}{l}\text { UP: cortisol; } \\
\text { DOWN: Glycerophospholipids, } \\
\text { Sphingolipids. }\end{array}$ \\
\hline Lautaoja et al. (2019) & Mice & Serum, Muscle & GC-MS & UP: phenylalanine. \\
\hline $\begin{array}{l}\text { Boguszewicz et al. } \\
\text { (2019) }\end{array}$ & Patients & Serum & NMR & UP: 3-hydroxybutyrate. \\
\hline Miller et al. (2019) & Patients & Plasma & LC-MS & $\begin{array}{l}\text { UP: IysoPC 18.2, L-proline, hexadecanoic acid, octadecanoic acid, phenylalanine } \\
\text { and lysoPC 16:1. }\end{array}$ \\
\hline Pin et al. (2019) & Mice & $\begin{array}{l}\text { Plasma, Muscle, } \\
\text { Liver }\end{array}$ & NMR, MS & $\begin{array}{l}\text { UP: low-density lipoprotein particles; } \\
\text { DOWN: circulating glucose, liver glucose and glycogen. }\end{array}$ \\
\hline Uzu et al. (2019) & Mice & Brain & CE-MS & Activated purine metabolism, Enhanced xanthine oxidase activity. \\
\hline Cui et al. (2019a) & Mice & $\begin{array}{l}\text { Tumor, } \\
\text { Serum, } \\
\text { Muscle }\end{array}$ & NMR & $\begin{array}{l}\text { UP (tumor): pyruvate and lactate; } \\
\text { DOWN (tumor): hypoxanthine, inosine, inosinate; } \\
\text { UP (serum): lactate and glycerol; } \\
\text { DOWN (serum): glucose; } \\
\text { UP (muscle): a-ketoglutarate; } \\
\text { DOWN (muscle): glucose. }\end{array}$ \\
\hline Cui et al. (2019b) & Mice & Muscle & NMR & $\begin{array}{l}\text { UP: glutamate, arginine, BCAAs; } \\
\text { DOWN: glucose, glycerol, } \\
\text { 3-hydroxybutyrate. }\end{array}$ \\
\hline Kunz et al. (2020) & Mice & $\begin{array}{l}\text { Plasma, } \\
\text { Muscle }\end{array}$ & LC-MS & UP: asymmetric dimethylarginine; and NG-monomethyl-L-arginine. \\
\hline Viana et al. (2020) & Rat & Serum & NMR & Promoted amino acid biosynthesis and metabolism. \\
\hline Miyaguti et al. (2020) & Rat & $\begin{array}{l}\text { Serum, } \\
\text { Muscle }\end{array}$ & NMR & UP: tryptophan, phenylalanine, histidine, glutamine. \\
\hline Chiocchetti et al. (2021) & Rat & Muscle & NMR & Increased amino acid levels and disordered energetic metabolism. \\
\hline Potgens et al. (2021) & Mice & $\begin{array}{l}\text { Caecal, portal vein, } \\
\text { liver, } \\
\text { vena cava }\end{array}$ & NMR & $\begin{array}{l}\text { Suppressed glycolysis and gluconeogenesis, hepatic carnitine and } \\
\text { phosphatidylcholine pathway activity; activated hexosamine pathway. }\end{array}$ \\
\hline Ni et al. (2021) & Patients & Plasma, Gut & LC-MS & DOWN: methylhistamine, BCAAs, vitamins. \\
\hline Ballaro et al. (2021) & Mice & $\begin{array}{l}\text { Muscle, Liver, } \\
\text { Plasma }\end{array}$ & NMR & $\begin{array}{l}\text { UP: glutamine, isoleucine, leucine, valine and phenylalanine; } \\
\text { DOWN: NADH and succinate. }\end{array}$ \\
\hline Sadek et al. (2021) & Mice & Muscle & $\begin{array}{l}\text { LC-MS } \\
\text { GC-MS }\end{array}$ & $\begin{array}{l}\text { UP: arginine, lysine, tryptophan, and methylhistidine; } \\
\text { DOWN: pyruvate, a-ketoglutarate and succinate. }\end{array}$ \\
\hline Zhou et al. (2021) & Mice & Muscle & NMR & Enhanced muscular proteolysis, suppressed glycolysis and ketone body oxidation. \\
\hline
\end{tabular}


On the other hand, Tseng and colleagues performed in-depth assessments of anti-cachectic activities of a novel histone deacetylase inhibitor AR-42 in C26 and LLC mouse models (Tseng et al., 2015). The LC-MS-based metabolomic analysis displayed that impaired glycolysis, glycogen synthesis and protein turnover in cachectic muscle tissues, could be improved by AR-42 and maintain the homeostatic metabolism relative to controls. Furthermore, Fukawa and colleagues conducted LC-MS-based metabolomic analysis integrated with transcriptomic analysis in muscles in a subcutaneous kidney murine model. They found that tumorsecreted factors induced excessive fatty acid oxidation, leading to muscle tissue dysfunction and activated p38 pathway. Afterwards, they indicated that drug inhibition of fatty acid oxidation could ameliorate human myotubes atrophy in vitro, and further restore muscle mass and body weight of mice in vivo (Fukawa et al., 2016). This work provided the inspiration on how to use non-targeted metabolomic techniques to explore new therapeutic targets. Initially, the researchers elucidated metabolic alterations mainly related to excessive fatty acid oxidation in animal models by performing metabolomics analysis. Then, they conducted pharmacological inhibition of fatty acid oxidation based on in vivo and in vitro models. Ultimately, they successfully rescued body weight loss and muscle atrophy in CC mice.

Recently, our group performed integrative NMR-based metabolomic and transcriptomic analyses of gastrocnemius in two murine models of CC (CT26 and LLC), and evaluated the beneficial effects of amiloride for CC treatments (Zhou et al., 2021). We identified significantly impaired metabolic pathways including enhanced muscular proteolysis, suppressed glycolysis and ketone body oxidation in cachectic gastrocnemius. Our results indicated that amiloride can alleviate muscle loss and the progression of $\mathrm{CC}$ through blocking exosome release originated from cancer cells. Our study suggests that tumorreleased exosome can be a potential target to attenuate muscle wasting during the progression of CC in the future.

In addition to the drug prevention, nutritional supplementation of metabolites such as BCAAs has been applied to improve impaired skeletal muscle metabolisms in diseases like AIDS and diabetes (Viana and GomesMarcondes, 2013). Furthermore, previous studies have demonstrated that leucine supplementation can promote nitrogen balance and restore muscle mass (Ventrucci et al., 2004; Salomao and Gomes-Marcondes, 2012). This team assessed if a leucine-rich diet could affect metabolic profiles of sera and tumor tissues in a rat model. The results exhibited downregulated levels of tryptophan and lactate associated with a suppressed hypermetabolic state, and up-regulated levels of $\beta$ hydroxybutyrate and acetoacetate, which might indirectly contribute to the prevention of CC (Viana et al., 2016). Recently, this group conducted metabolomic analyses of sera and gastrocnemius derived from rats with leucine supplementation. The tumor-bearing rats displayed distinctly altered metabolic pathways including protein biosynthesis, glycine, serine and threonine metabolism, and ammonia recycling. Significantly, the leucine-rich diet rats showed attenuated Warburg effect and improved lipid metabolism (Miyaguti et al., 2020).

Ketone body supplementation might also contribute to regulation of glucose and lipid metabolism and prevent body weight loss (Kennedy et al., 2007). Shukla and colleagues addressed anti-cancerous and anti-cachectic properties of a ketogenic diet in vitro, and assessed the effects of ketone bodies on tumor mass and CC symptoms of mice by conducting NMR-based metabolomic analysis in vivo (Shukla et al., 2014). They observed reduced glycolytic flux and diminished glutamine uptake, decreased overall ATP content in tumor cells. These results suggest that treatment with ketone bodies could prevent cachexia phenotype. Collectively, we anticipate that exploitation of the global metabolome with metabolomics techniques can achieve more comprehensive knowledge of CC and discover effective therapeutic strategies.

\section{CONCLUSION}

Even though metabolomics is relatively less used compared with other omics approaches, it is able to provide key information for further exploration of CC, including mechanistic understanding, potential biomarkers, metabolic signatures, and therapeutic strategies. With the rapid development and wide application of metabolomics analysis in the field of CC research, in-depth understandings of CC have been broadly expanded and systemized. Researchers propose novel hypotheses and develop approaches using metabolomic techniques, to exploit the features of CC and therapeutic targets for the treatments of CC. Metabolomics can be employed to identify potential biomarkers for screening early symptoms and monitoring the progression of CC, through measuring alterations in concentrations of hundreds of endogenous metabolites in biofluids and tissues derived from animal and human beings. In addition, numerous studies have shown that targeting specific metabolic pathways could regulate abnormal metabolisms induced by CC and ultimately alleviate syndromes of CC. Table 2 displays the summary of the metabolomics studies on $\mathrm{CC}$ in the past decade with novel discoveries.

Current studies indicate that the metabolites of carbohydrates, lipids and amino acids are closely linked to the development and progression of CC (Figure 2). Carbohydrates related to CC primarily include glucose and lactate, TCA cycle metabolites such as citrate, succinate, and $\alpha$-ketoglutarate. Lipids relevant to CC mainly include glycerophospholipids, LDL and lipid derivatives. Amino acids participating in the pathogenesis of CC mostly include BCAAs, phenylalanine and their metabolites. In addition, three kinds of ketone bodies and methylhistidine and its metabolites are also important substances involved in the pathological mechanisms of CC.

Significantly impaired metabolic pathways are associated with the pathogenesis of CC, including two main types: energy metabolism and amino acid metabolism (Figure 2). The metabolism of amino acids is usually disordered in CC mainly due to muscle turnover imbalance, such as BCAAs metabolism, arginine metabolism, glutamate and glutamine metabolism, 
phenylalanine and tyrosine metabolism. Glycolysis, fatty acid oxidation, and TCA cycle are mostly disturbed because of the shifted energy needs. Additionally, impaired metabolisms of carbohydrates and lipids contribute to the progression of CC via a series of metabolic pathways.

\section{FUTURE PERSPECTIVES}

Although the metabolic signatures of cachectic muscle are being investigated in the past decade, we still need to know how to elucidate the molecular mechanisms based on the results obtained from metabolomic analyses. The chemical complexity and large number of metabolites might be one of the challenges associated with metabolomic analyses. For example, metabolite compositions of sera, plasma and urine are manifestations of tumor, liver, muscle, and functions of gut microbes, type of diets, clinical cancer treatment, and other tumor-derived factors like exosomes and cytokines. Compared with other omics approaches, metabolomics has many advantages and also some drawbacks. No techniques are really flawless as a fact. Expectedly, metabolomic analyses should be integrated with other omics approaches, bioinformatics, biophysical techniques and signaling pathway analysis, which would provide

\section{REFERENCES}

Adams, V., Späte, U., Kränkel, N., Schulze, P. C., Linke, A., Schuler, G., et al. (2003). Nuclear Factor-Kappa B Activation in Skeletal Muscle of Patients with Chronic Heart Failure: Correlation with the Expression of Inducible Nitric Oxide Synthase. Eur. J. Cardiovasc. Prev. Rehabil. 10 (4), 273-277. doi:10.1097/ 00149831-200308000-00009

Agusti, A., Morla, M., Sauleda, J., Saus, C., and Busquets, X. (2004). NF- B Activation and iNOS Upregulation in Skeletal Muscle of Patients with COPD and Low Body Weight. Thorax 59 (6), 483-487. doi:10.1136/thx. 2003.017640

Argilés, J. M., López-Soriano, F. J., and Busquets, S. (2013). Mechanisms and Treatment of Cancer Cachexia. Nutr. Metab. Cardiovasc. Dis. 23 (Suppl. 1), S19-S24. doi:10.1016/j.numecd.2012.04.011

Argilés, J. M., and Stemmler, B. (2013). The Potential of Ghrelin in the Treatment of Cancer Cachexia. Expert Opin. Biol. Ther. 13 (1), 67-76. doi:10.1517/ 14712598.2013.727390

Ballarò, R., Lopalco, P., Audrito, V., Beltrà, M., Pin, F., Angelini, R., et al. (2021). Targeting Mitochondria by SS-31 Ameliorates the Whole Body Energy Status in Cancer- and Chemotherapy-Induced Cachexia. Cancers 13 (4), 850. doi:10. 3390/cancers 13040850

Beckonert, O., Keun, H. C., Ebbels, T. M. D., Bundy, J., Holmes, E., Lindon, J. C., et al. (2007). Metabolic Profiling, Metabolomic and Metabonomic Procedures for NMR Spectroscopy of Urine, Plasma, Serum and Tissue Extracts. Nat. Protoc. 2 (11), 2692-2703. doi:10.1038/nprot.2007.376

B. Heymsfield, S., Wang, Z., Baumgartner, R. N., and Ross, R. (1997). Human Body Composition: Advances in Models and Methods. Annu. Rev. Nutr. 17, 527-558. doi:10.1146/annurev.nutr.17.1.527

Bindels, L. B., Beck, R., Schakman, O., Martin, J. C., De Backer, F., Sohet, F. M., et al. (2012). Restoring Specific Lactobacilli Levels Decreases Inflammation and Muscle Atrophy Markers in an Acute Leukemia Mouse Model. PLoS One 7 (6), e37971. doi:10.1371/journal.pone.0037971

Bindels, L. B., Neyrinck, A. M., Claus, S. P., Le Roy, C. I., Grangette, C., Pot, B., et al. (2016). Synbiotic Approach Restores Intestinal Homeostasis and Prolongs Survival in Leukaemic Mice with Cachexia. ISME J. 10 (6), 1456-1470. doi:10.1038/ismej.2015.209 comprehensive views on the complicated pathogenesis of CC, and expand our knowledge of fundamental mechanisms underlying metabolic disorder and muscle wasting. As unified workflows, inexpensive equipment, and humanized acquisition software and high throughput measurements as well as powerful computational analysis become more broadly available, metabolomics will play increasingly vital roles in the studies of molecular biosciences.

\section{AUTHOR CONTRIBUTIONS}

The conception and design were performed by $\mathrm{PC}, \mathrm{CH}$, and DL. The manuscript was drafted by PC. The manuscript was discussed and revised by PC, XL, QL, CH, and DL. All authors read and approved the final manuscript.

\section{FUNDING}

This work was supported by the National Natural Science Foundation of China (No. 31971357) and the Open Research Fund of State Key Laboratory of Cellular Stress Biology, Xiamen University (SKLCSB2020KF002).

Blum, D., Omlin, A., Omlin, A., Fearon, K., Baracos, V., Radbruch, L., et al. (2010). Evolving Classification Systems for Cancer Cachexia: Ready for Clinical Practice? Support Care Cancer 18 (3), 273-279. doi:10.1007/s00520-009-0800-6

Bodine, S. C., Stitt, T. N., Gonzalez, M., Kline, W. O., Stover, G. L., Bauerlein, R., et al. (2001). Akt/mTOR Pathway Is a Crucial Regulator of Skeletal Muscle Hypertrophy and Can Prevent Muscle Atrophy In Vivo. Nat. Cel Biol 3 (11), 1014-1019. doi:10.1038/ncb1101-1014

Boguszewicz, Ł., Bieleń, A., Mrochem-Kwarciak, J., Skorupa, A., Ciszek, M., Heyda, A., et al. (2019). NMR-based Metabolomics in Real-Time Monitoring of Treatment Induced Toxicity and Cachexia in Head and Neck Cancer: a Method for Early Detection of High Risk Patients. Metabolomics 15 (8), 110. doi:10.1007/s11306-019-1576-4

Bonaldo, P., and Sandri, M. (2013). Cellular and Molecular Mechanisms of Muscle Atrophy. Dis. Model. Mech. 6 (1), 25-39. doi:10.1242/dmm.010389

Bothwell, J. H. F., and Griffin, J. L. (2011). An Introduction to Biological Nuclear Magnetic Resonance Spectroscopy. Biol. Rev. Camb Philos. Soc. 86 (2), 493-510. doi:10.1111/j.1469-185X.2010.00157.x

Bozzetti, F., and Mariani, L. (2009). Defining and Classifying Cancer Cachexia: a Proposal by the SCRINIO Working Group. JPEN J. Parenter. Enteral Nutr. 33 (4), 361-367. doi:10.1177/0148607108325076

Cala, M. P., Agulló-Ortuño, M. T., Prieto-García, E., González-Riano, C., ParrillaRubio, L., Barbas, C., et al. (2018). Multiplatform Plasma Fingerprinting in Cancer Cachexia: a Pilot Observational and Translational Study. J. Cachexia, Sarcopenia Muscle 9 (2), 348-357. doi:10.1002/jcsm.12270

Chiocchetti, G. d. M. e., Lopes-Aguiar, L., Miyaguti, N. A. d. S., Viana, L. R., Salgado, C. d. M., Orvoën, O. O., et al. (2021). A Time-Course Comparison of Skeletal Muscle Metabolomic Alterations in Walker-256 Tumour-Bearing Rats at Different Stages of Life. Metabolites 11 (6), 404. doi:10.3390/metabo11060404

Cui, P., Huang, C., Guo, J., Wang, Q., Liu, Z., Zhuo, H., et al. (2019a). Metabolic Profiling of Tumors, Sera, and Skeletal Muscles from an Orthotopic Murine Model of Gastric Cancer Associated-Cachexia. J. Proteome Res. 18 (4), 1880-1892. doi:10.1021/acs.jproteome.9b00088

Cui, P., Shao, W., Huang, C., Wu, C.-J., Jiang, B., and Lin, D. (2019b). Metabolic Derangements of Skeletal Muscle from a Murine Model of Glioma Cachexia. Skeletal Muscle 9 (1), 3. doi:10.1186/s13395-018-0188-4

Deans, D. A. C., Tan, B. H., Wigmore, S. J., Ross, J. A., de Beaux, A. C., PatersonBrown, S., et al. (2009). The Influence of Systemic Inflammation, Dietary Intake 
and Stage of Disease on Rate of Weight Loss in Patients with GastroOesophageal Cancer. Br. J. Cancer 100 (1), 63-69. doi:10.1038/sj.bjc.6604828

Der-Torossian, H., Asher, S. A., Winnike, J. H., Wysong, A., Yin, X., Willis, M. S., et al. (2013a). Cancer Cachexia's Metabolic Signature in a Murine Model Confirms a Distinct Entity. Metabolomics 9 (3), 730-739. doi:10.1007/s11306012-0485-6

Der-Torossian, H., Wysong, A., Shadfar, S., Willis, M. S., McDunn, J., and Couch, M. E. (2013b). Metabolic Derangements in the Gastrocnemius and the Effect of Compound A Therapy in a Murine Model of Cancer Cachexia. J. Cachexia Sarcopenia Muscle 4 (2), 145-155. doi:10.1007/s13539-012-0101-7

Diffee, G. M., Kalfas, K., Al-Majid, S., and McCarthy, D. O. (2002). Altered Expression of Skeletal Muscle Myosin Isoforms in Cancer Cachexia. Am. J. Physiology-Cell Physiol. 283 (5), C1376-C1382. doi:10.1152/ajpcell.00154. 2002

Dunn, W. B., Broadhurst, D. I., Atherton, H. J., Goodacre, R., and Griffin, J. L. (2011). Systems Level Studies of Mammalian Metabolomes: the Roles of Mass Spectrometry and Nuclear Magnetic Resonance Spectroscopy. Chem. Soc. Rev. 40 (1), 387-426. doi:10.1039/b906712b

Eisner, R., Stretch, C., Eastman, T., Xia, J., Hau, D., Damaraju, S., et al. (2011). Learning to Predict Cancer-Associated Skeletal Muscle Wasting from 1H-NMR Profiles of Urinary Metabolites. Metabolomics 7 (1), 25-34. doi:10.1007/ s11306-010-0232-9

Emwas, A.-H. M. (2015). The Strengths and Weaknesses of NMR Spectroscopy and Mass Spectrometry with Particular Focus on Metabolomics Research. Methods Mol. Biol. 1277, 161-193. doi:10.1007/978-1-4939-2377-9_13

Evans, W. J., Morley, J. E., Argilés, J., Bales, C., Baracos, V., Guttridge, D., et al. (2008). Cachexia: A New Definition. Clin. Nutr. 27 (6), 793-799. doi:10.1016/j. clnu.2008.06.013

Evans, W. J. (2010). Skeletal Muscle Loss: Cachexia, Sarcopenia, and Inactivity. Am. J. Clin. Nutr. 91 (4), 1123S-1127S. doi:10.3945/ajen.2010.28608A

Fearon, K. C. H. (1992). The Mechanisms and Treatment of Weight Loss in Cancer. Proc. Nutr. Soc. 51 (2), 251-265. doi:10.1079/pns19920036

Fearon, K., Strasser, F., Anker, S. D., Bosaeus, I., Bruera, E., Fainsinger, R. L., et al. (2011). Definition and Classification of Cancer Cachexia: an International Consensus. Lancet Oncol. 12 (5), 489-495. doi:10.1016/S1470-2045(10)70218-7

Fiehn, O., Kopka, J., Dörmann, P., Altmann, T., Trethewey, R. N., and Willmitzer, L. (2000). Metabolite Profiling for Plant Functional Genomics. Nat. Biotechnol. 18 (11), 1157-1161. doi:10.1038/81137

Fujiwara, Y., Kobayashi, T., Chayahara, N., Imamura, Y., Toyoda, M., Kiyota, N., et al. (2014). Metabolomics Evaluation of Serum Markers for Cachexia and Their Intra-day Variation in Patients with Advanced Pancreatic Cancer. PLoS One 9 (11), el13259. doi:10.1371/journal.pone.0113259

Fukawa, T., Yan-Jiang, B. C., Min-Wen, J. C., Jun-Hao, E. T., Huang, D., Qian, C.N., et al. (2016). Excessive Fatty Acid Oxidation Induces Muscle Atrophy in Cancer Cachexia. Nat. Med. 22 (6), 666-671. doi:10.1038/nm.4093

Gallagher, I. J., Jacobi, C., Tardif, N., Rooyackers, O., and Fearon, K. (2016). Omics/ systems Biology and Cancer Cachexia. Semin. Cel Develop. Biol. 54, 92-103. doi:10.1016/j.semcdb.2015.12.022

Gao, H., Lu, Q., Liu, X., Cong, H., Zhao, L., Wang, H., et al. (2009). Application of1H NMR-Based Metabonomics in the Study of Metabolic Profiling of Human Hepatocellular Carcinoma and Liver Cirrhosis. Cancer Sci. 100 (4), 782-785. doi:10.1111/j.1349-7006.2009.01086.x

Gupta, S. C., Kim, J. H., Kannappan, R., Reuter, S., Dougherty, P. M., and Aggarwal, B. B. (2011). Role of Nuclear Factor-Kb-Mediated Inflammatory Pathways in Cancer-Related Symptoms and Their Regulation by Nutritional Agents. Exp. Biol. Med. (Maywood) 236 (6), 658-671. doi:10.1258/ebm.2011.011028

Halle, J. L., Counts, B. R., and Carson, J. A. (2020). Exercise as a Therapy for Cancer-Induced Muscle Wasting. Sports Med. Health Sci. 2 (4), 186-194. doi:10. 1016/j.smhs.2020.11.004

Hamerman, D. (2002). Molecular-based Therapeutic Approaches in Treatment of Anorexia of Aging and Cancer Cachexia. Journals Gerontol. Ser. A: Biol. Sci. Med. Sci. 57 (8), M511-M518. doi:10.1093/gerona/57.8.m511

Idborg-Björkman, H., Edlund, P.-O., Kvalheim, O. M., Schuppe-Koistinen, I., and Jacobsson, S. P. (2003). Screening of Biomarkers in Rat Urine Using LC/ electrospray Ionization-MS and Two-Way Data Analysis. Anal. Chem. 75 (18), 4784-4792. doi:10.1021/ac0341618

Jonsson, P., Bruce, S. J., Moritz, T., Trygg, J., Sjöström, M., Plumb, R., et al. (2005). Extraction, Interpretation and Validation of Information for Comparing
Samples in Metabolic LC/MS Data Sets. Analyst 130 (5), 701-707. doi:10. 1039/b501890k

Kennedy, A. R., Pissios, P., Otu, H., Xue, B., Asakura, K., Furukawa, N., et al. (2007). A High-Fat, Ketogenic Diet Induces a Unique Metabolic State in Mice. Am. J. Physiology-Endocrinology Metab. 292 (6), E1724-E1739. doi:10.1152/ ajpendo.00717.2006

Kumar, N. B., Kazi, A., Smith, T., Crocker, T., Yu, D., Reich, R. R., et al. (2010). Cancer Cachexia: Traditional Therapies and Novel Molecular MechanismBased Approaches to Treatment. Curr. Treat. Options. Oncol. 11 (3-4), 107-117. doi:10.1007/s11864-010-0127-z

Kunz, H. E., Dorschner, J. M., Berent, T. E., Meyer, T., Wang, X., Jatoi, A., et al. (2020). Methylarginine Metabolites Are Associated with Attenuated Muscle Protein Synthesis in Cancer-Associated Muscle Wasting. J. Biol. Chem. 295 (51), 17441-17459. doi:10.1074/jbc.RA120.014884

Lallukka, S., and Yki-Järvinen, H. (2016). Non-alcoholic Fatty Liver Disease and Risk of Type 2 Diabetes. Best Pract. Res. Clin. Endocrinol. Metab. 30 (3), 385-395. doi:10.1016/j.beem.2016.06.006

Lautaoja, J. H., Lalowski, M., Nissinen, T. A., Hentilä, J., Shi, Y., Ritvos, O., et al. (2019). Muscle and Serum Metabolomes Are Dysregulated in colon-26 TumorBearing Mice Despite Amelioration of Cachexia with Activin Receptor Type 2B Ligand Blockade. Am. J. Physiology-Endocrinology Metab. 316 (5), E852-E865. doi:10.1152/ajpendo.00526.2018

Lee, S.-J. (2004). Regulation of Muscle Mass by Myostatin. Annu. Rev. Cel Dev. Biol. 20, 61-86. doi:10.1146/annurev.cellbio.20.012103.135836

Legido-Quigley, C., Stella, C., Perez-Jimenez, F., Lopez-Miranda, J., Ordovas, J., Powell, J., et al. (2010). Liquid Chromatography-Mass Spectrometry Methods for Urinary Biomarker Detection in Metabonomic Studies with Application to Nutritional Studies. Biomed. Chromatogr. 24 (7), 737-743. doi:10.1002/bmc. 1357

Li, A.-P., Li, Z.-Y., Sun, H.-F., Li, K., Qin, X.-M., and Du, G.-H. (2015). Comparison of Two Different Astragali Radix by a 1H NMR-Based Metabolomic Approach. J. Proteome Res. 14 (5), 2005-2016. doi:10.1021/pr501167u

Lim, S., Brown, J. L., Washington, T. A., and Greene, N. P. (2020). Development and Progression of Cancer Cachexia: Perspectives from Bench to Bedside. Sports Med. Health Sci. 2 (4), 177-185. doi:10.1016/j.smhs.2020.10.003

Loberg, R. D., Bradley, D. A., Tomlins, S. A., Chinnaiyan, A. M., and Pienta, K. J. (2007). The Lethal Phenotype of Cancer: The Molecular Basis of Death Due to Malignancy. CA: A Cancer J. Clinicians 57 (4), 225-241. doi:10.3322/canjclin. 57.4.225

Lok, C. (2015). Cachexia: The Last Illness. Nature 528 (7581), 182-183. doi:10. 1038/528182a

Ma, Q., Li, Y., Wang, M., Tang, Z., Wang, T., Liu, C., et al. (2018). Progress in Metabonomics of Type 2 Diabetes Mellitus. Molecules 23 (7), 1834. doi:10.3390/ molecules 23071834

Miller, J., Alshehri, A., Ramage, M. I., Stephens, N. A., Mullen, A. B., Boyd, M., et al. (2019). Plasma Metabolomics Identifies Lipid and Amino Acid Markers of Weight Loss in Patients with Upper Gastrointestinal Cancer. Cancers 11 (10), 1594. doi:10.3390/cancers 11101594

Mitsiopoulos, N., Baumgartner, R. N., Heymsfield, S. B., Lyons, W., Gallagher, D., and Ross, R. (19981985). Cadaver Validation of Skeletal Muscle Measurement by Magnetic Resonance Imaging and Computerized Tomography. J. Appl. Physiol. 85 (1), 115-122. doi:10.1152/jappl.1998.85.1.115

Miyaguti, N. A. d. S., Stanisic, D., Oliveira, S. C. P. d., Dos Santos, G. S., Manhe, B. S., Tasic, L., et al. (2020). Serum and Muscle 1H NMR-Based Metabolomics Profiles Reveal Metabolic Changes Influenced by a Maternal Leucine-Rich Diet in TumorBearing Adult Offspring Rats. Nutrients 12 (7), 2106. doi:10.3390/nu12072106

Mourtzakis, M., Prado, C. M. M., Lieffers, J. R., Reiman, T., McCargar, L. J., and Baracos, V. E. (2008). A Practical and Precise Approach to Quantification of Body Composition in Cancer Patients Using Computed Tomography Images Acquired during Routine Care. Appl. Physiol. Nutr. Metab. 33 (5), 997-1006. doi:10.1139/H08-075

Muroya, S., Ueda, S., Komatsu, T., Miyakawa, T., and Ertbjerg, P. (2020). MEATabolomics: Muscle and Meat Metabolomics in Domestic Animals. Metabolites 10 (5), 188. doi:10.3390/metabo10050188

Musarò, A., McCullagh, K., Paul, A., Houghton, L., Dobrowolny, G., Molinaro, M., et al. (2001). Localized Igf-1 Transgene Expression Sustains Hypertrophy and Regeneration in Senescent Skeletal Muscle. Nat. Genet. 27 (2), 195-200. doi:10. $1038 / 84839$ 
Nagaya, N., Kojima, M., and Kangawa, K. (2006). Ghrelin, a Novel Growth Hormonereleasing Peptide, in the Treatment of Cardiopulmonaryassociated Cachexia. Intern. Med. 45 (3), 127-134. doi:10.2169/internalmedicine.45.1402

Newgard, C. B., An, J., Bain, J. R., Muehlbauer, M. J., Stevens, R. D., Lien, L. F., et al. (2009). A Branched-Chain Amino Acid-Related Metabolic Signature that Differentiates Obese and Lean Humans and Contributes to Insulin Resistance. Cel Metab. 9 (4), 311-326. doi:10.1016/j.cmet.2009.02.002

Newgard, C. B. (2017). Metabolomics and Metabolic Diseases: Where Do We Stand? Cel Metab. 25 (1), 43-56. doi:10.1016/j.cmet.2016.09.018

Ni, Y., Lohinai, Z., Heshiki, Y., Dome, B., Moldvay, J., Dulka, E., et al. (2021). Distinct Composition and Metabolic Functions of Human Gut Microbiota Are Associated with Cachexia in Lung Cancer Patients. ISME J. 15, 3207-3220. doi:10.1038/s41396-021-00998-8

Nicholson, J. K., Lindon, J. C., and Holmes, E. (1999). 'Metabonomics': Understanding the Metabolic Responses of Living Systems to Pathophysiological Stimuli via Multivariate Statistical Analysis of Biological NMR Spectroscopic Data. Xenobiotica 29 (11), 1181-1189. doi:10.1080/ 004982599238047

Nikolic, S. B., Sharman, J. E., Adams, M. J., and Edwards, L. M. (2014). Metabolomics in Hypertension. J. Hypertens. 32 (6), 1159-1169. doi:10. 1097/HJH.0000000000000168

O’Connell, T. M., Ardeshirpour, F., Asher, S. A., Winnike, J. H., Yin, X., George, J., et al. (2008). Metabolomic Analysis of Cancer Cachexia Reveals Distinct Lipid and Glucose Alterations. Metabolomics 4 (3), 216-225. doi:10.1007/s11306008-0113-7

Ohbuchi, K., Nishiumi, S., Fujitsuka, N., Hattori, T., Yamamoto, M., Inui, A., et al. (2015). Rikkunshito Ameliorates Cancer Cachexia Partly through Elevation of Glucarate in Plasma. Evidence-Based Complement. Altern. Med. 2015, 1-11. doi:10.1155/2015/871832

Pang, Z., Chong, J., Zhou, G., de Lima Morais, D. A., Chang, L., Barrette, M., et al. (2021). MetaboAnalyst 5.0: Narrowing the gap between Raw Spectra and Functional Insights. Nucleic Acids Res. 49 (W1), W388-W396. doi:10.1093/ nar/gkab382

Penna, F., Ballarò, R., Martinez-Cristobal, P., Sala, D., Sebastian, D., Busquets, S., et al. (2019). Autophagy Exacerbates Muscle Wasting in Cancer Cachexia and Impairs Mitochondrial Function. J. Mol. Biol. 431 (15), 2674-2686. doi:10.1016/ j.jmb.2019.05.032

Peterson, J. M., Bakkar, N., and Guttridge, D. C. (2011). NF-кB Signaling in Skeletal Muscle Health and Disease. Curr. Top. Dev. Biol. 96, 85-119. doi:10.1016/B9780-12-385940-2.00004-8

Pin, F., Barreto, R., Couch, M. E., Bonetto, A., and O’Connell, T. M. (2019). Cachexia Induced by Cancer and Chemotherapy Yield Distinct Perturbations to Energy Metabolism. J. Cachexia, Sarcopenia Muscle 10 (1), 140-154. doi:10. $1002 /$ jcsm. 12360

Pötgens, S. A., Thibaut, M. M., Joudiou, N., Sboarina, M., Neyrinck, A. M., Cani, P. D., et al. (2021). Multi-compartment Metabolomics and Metagenomics Reveal Major Hepatic and Intestinal Disturbances in Cancer Cachectic Mice. J. Cachexia, Sarcopenia Muscle 12 (2), 456-475. doi:10.1002/jcsm.12684

Quan-Jun, Y., Jun, B., Li-Li, W., Yong-Long, H., Bin, L., Qi, Y., et al. (2015). NMRbased Metabolomics Reveals Distinct Pathways Mediated by Curcumin in Cachexia Mice Bearing CT26 Tumor. RSC Adv. 5 (16), 11766-11775. doi:10. 1039/c4ra14128h

Quanjun, Y., Genjin, Y., Lili, W., Bin, L., Jin, L., Qi, Y., et al. (2013). Serum Metabolic Profiles Reveal the Effect of Formoterol on Cachexia in TumorBearing Mice. Mol. Biosyst. 9 (12), 3015-3025. doi:10.1039/c3mb70134d

QuanJun, Y., GenJin, Y., LiLi, W., Yan, H., YongLong, H., Jin, L., et al. (2015). Integrated Analysis of Serum and Intact Muscle Metabonomics Identify Metabolic Profiles of Cancer Cachexia in a Dynamic Mouse Model. RSC Adv. 5 (112), 92438-92448. doi:10.1039/c5ra19004e

Ramamoorthy, S., Donohue, M., and Buck, M. (2009). Decreased Jun-D and Myogenin Expression in Muscle Wasting of Human Cachexia. Am. J. Physiology-Endocrinology Metab. 297 (2), E392-E401. doi:10.1152/ ajpendo.90529.2008

Ramautar, R., Somsen, G. W., and de Jong, G. J. (2019). CE-MS for Metabolomics: Developments and Applications in the Period 2016-2018. Electrophoresis 40 (1), 165-179. doi:10.1002/elps.201800323

Rohm, M., Zeigerer, A., Machado, J., and Herzig, S. (2019). Energy Metabolism in Cachexia. EMBO Rep. 20 (4). doi:10.15252/embr.201847258
Rolland, Y., Van Kan, G. A., Gillette-Guyonnet, S., and Vellas, B. (2011). Cachexia versus Sarcopenia. Curr. Opin. Clin. Nutr. Metab. Care 14 (1), 15-21. doi:10. 1097/MCO.0b013e328340c2c2

Römisch-Margl, W., Prehn, C., Bogumil, R., Röhring, C., Suhre, K., and Adamski, J. (2011). Procedure for Tissue Sample Preparation and Metabolite Extraction for High-Throughput Targeted Metabolomics. Metabolomics 8 (1), 133-142. doi:10.1007/s11306-011-0293-4

Sadek, J., Hall, D. T., Colalillo, B., Omer, A., Tremblay, A. M. K., SanguinGendreau, V., et al. (2021). Pharmacological or Genetic Inhibition of iNOS Prevents Cachexia-mediated Muscle Wasting and its Associated Metabolism Defects. EMBO Mol. Med. 13 (7), e13591. doi:10.15252/emmm.202013591

Salomão, E. M., and Gomes-Marcondes, M. C. C. (2012). Light Aerobic Physical Exercise in Combination with Leucine And/or Glutamine-Rich Diet Can Improve the Body Composition and Muscle Protein Metabolism in Young Tumor-Bearing Rats. J. Physiol. Biochem. 68 (4), 493-501. doi:10.1007/s13105012-0164-0

Sandri, M., Sandri, C., Gilbert, A., Skurk, C., Calabria, E., Picard, A., et al. (2004). Foxo Transcription Factors Induce the Atrophy-Related Ubiquitin Ligase Atrogin-1 and Cause Skeletal Muscle Atrophy. Cell 117 (3), 399-412. doi:10. 1016/s0092-8674(04)00400-3

Santarpia, L., Contaldo, F., and Pasanisi, F. (2011). Nutritional Screening and Early Treatment of Malnutrition in Cancer Patients. J. Cachexia Sarcopenia Muscle 2 (1), 27-35. doi:10.1007/s13539-011-0022-x

Schmidt, D. R., Patel, R., Kirsch, D. G., Lewis, C. A., Vander Heiden, M. G., and Locasale, J. W. (2021). Metabolomics in Cancer Research and Emerging Applications in Clinical Oncology. CA A. Cancer J. Clin. 71 (4), 333-358. doi: $10.3322 /$ caac. 21670

Schwämmle, V., Verano-Braga, T., and Roepstorff, P. (2015). Computational and Statistical Methods for High-Throughput Analysis of post-translational Modifications of Proteins. J. Proteomics 129, 3-15. doi:10.1016/j.jprot.2015. 07.016

Shah, S. H., Kraus, W. E., and Newgard, C. B. (2012a). Metabolomic Profiling for the Identification of Novel Biomarkers and Mechanisms Related to Common Cardiovascular Diseases. Circulation 126 (9), 1110-1120. doi:10.1161/ CIRCULATIONAHA.111.060368

Shah, S. H., Sun, J.-L., Stevens, R. D., Bain, J. R., Muehlbauer, M. J., Pieper, K. S., et al. (2012b). Baseline Metabolomic Profiles Predict Cardiovascular Events in Patients at Risk for Coronary Artery Disease. Am. Heart J. 163 (5), 844-850. e841. doi:10.1016/j.ahj.2012.02.005

Shen, W., Punyanitya, M., Wang, Z., Gallagher, D., St.-Onge, M.-P., Albu, J., et al. (20041985). Total Body Skeletal Muscle and Adipose Tissue Volumes: Estimation from a Single Abdominal Cross-Sectional Image. J. Appl. Physiol. 97 (6), 2333-2338. doi:10.1152/japplphysiol.00744.2004

Shukla, S. K., Gebregiworgis, T., Purohit, V., Chaika, N. V., Gunda, V., Radhakrishnan, P., et al. (2014). Metabolic Reprogramming Induced by Ketone Bodies Diminishes Pancreatic Cancer Cachexia. Cancer Metab. 2, 18. doi:10.1186/2049-3002-2-18

Stolz, A., Jooss, K., Höcker, O., Römer, J., Schlecht, J., and Neusüß, C. (2019). Recent Advances in Capillary Electrophoresis-Mass Spectrometry: Instrumentation, Methodology and Applications. Electrophoresis 40 (1), 79-112. doi:10.1002/elps.201800331

Stretch, C., Eastman, T., Mandal, R., Eisner, R., Wishart, D. S., Mourtzakis, M., et al. (2012). Prediction of Skeletal Muscle and Fat Mass in Patients with Advanced Cancer Using a Metabolomic Approach. J. Nutr. 142 (1), 14-21. doi:10.3945/jn.111.147751

Sugimoto, M., Kawakami, M., Robert, M., Soga, T., and Tomita, M. (2012). Bioinformatics Tools for Mass Spectroscopy-Based Metabolomic Data Processing and Analysis. Cbio 7 (1), 96-108. doi:10.2174/157489312799304431

Tisdale, M. J. (2003). Pathogenesis of Cancer Cachexia. J. Support. Oncol. 1 (3), 159-168.

Tisdale, M. J. (2009). Mechanisms of Cancer Cachexia. Physiol. Rev. 89 (2), 381-410. doi:10.1152/physrev.00016.2008

Tseng, Y.-C., Kulp, S. K., Lai, I.-L., Hsu, E.-C., He, W. A., Frankhouser, D. E., et al. (2015). Preclinical Investigation of the Novel Histone Deacetylase Inhibitor AR-42 in the Treatment of Cancer-Induced Cachexia. JNCI.J 107 (12), djv274. doi:10.1093/jnci/djv274

Tsiropoulou, S., McBride, M., and Padmanabhan, S. (2017). Urine Metabolomics in Hypertension Research. Methods Mol. Biol. 1527, 61-68. doi:10.1007/978-14939-6625-7_5 
Twelkmeyer, B., Tardif, N., and Rooyackers, O. (2017). Omics and Cachexia. Curr. Opin. Clin. Nutr. Metab. Care 20 (3), 181-185. doi:10.1097/Mco. 0000000000000363

Uzu, M., Nonaka, M., Miyano, K., Sato, H., Kurebayashi, N., Yanagihara, K., et al. (2019). A Novel Strategy for Treatment of Cancer Cachexia Targeting Xanthine Oxidase in the Brain. J. Pharmacol. Sci. 140 (1), 109-112. doi:10.1016/j.jphs. 2019.04.005

Valdes, A. M., Walter, J., Segal, E., and Spector, T. D. (2018). Role of the Gut Microbiota in Nutrition and Health. BMJ 361, k2179. doi:10.1136/bmj.k2179

Ventrucci, G., Ramos Silva, L. G., Roston Mello, M. A., and Gomes Marcondes, M. C. C. (2004). Effects of a Leucine-Rich Diet on Body Composition during Nutritional Recovery in Rats. Nutrition 20 (2), 213-217. doi:10.1016/j.nut.2003. 10.014

Viana, L. R., Canevarolo, R., Luiz, A. C. P., Soares, R. F., Lubaczeuski, C., Zeri, A. C. d. M., et al. (2016). Leucine-rich Diet Alters the 1H-NMR Based Metabolomic Profile without Changing the Walker-256 Tumour Mass in Rats. BMC Cancer 16 (1), 764. doi:10.1186/s12885-016-2811-2

Viana, L. R., and Gomes-Marcondes, M. C. C. (2013). Leucine-Rich Diet Improves the Serum Amino Acid Profile and Body Composition of Fetuses from TumorBearing Pregnant Mice1. Biol. Reprod. 88 (5), 121. doi:10.1095/biolreprod.112. 107276

Viana, L. R., Lopes-Aguiar, L., Rossi Rosolen, R., Willians Dos Santos, R., and Cintra Gomes-Marcondes, M. C. (2020). 1H-NMR Based Serum Metabolomics Identifies Different Profile between Sarcopenia and Cancer Cachexia in Ageing Walker 256 Tumour-Bearing RatsH-NMR Based Serum Metabolomics Identifies Different Profile between Sarcopenia and Cancer Cachexia in Ageing Walker 256 Tumour-Bearing Rats. Metabolites 10 (4), 161. doi:10. 3390/metabo10040161

Waddell, D. S., Baehr, L. M., van den Brandt, J., Johnsen, S. A., Reichardt, H. M., Furlow, J. D., et al. (2008). The Glucocorticoid Receptor and FOXO1 Synergistically Activate the Skeletal Muscle Atrophy-Associated MuRF1 Gene. Am. J. Physiology-Endocrinology Metab. 295 (4), E785-E797. doi:10. 1152/ajpendo.00646.2007

Wiklund, S., Johansson, E., Sjöström, L., Mellerowicz, E. J., Edlund, U., Shockcor, J. P., et al. (2008). Visualization of GC/TOF-MS-based Metabolomics Data for Identification of Biochemically Interesting Compounds Using OPLS Class Models. Anal. Chem. 80 (1), 115-122. doi:10.1021/ac0713510

Wishart, D. S. (2016). Emerging Applications of Metabolomics in Drug Discovery and Precision Medicine. Nat. Rev. Drug Discov. 15 (7), 473-484. doi:10.1038/ $\operatorname{nrd} .2016 .32$
Xia, F., and Wan, J. B. (2021). Chemical Derivatization Strategy for Mass Spectrometry-based Lipidomics. Mass. Spec. Rev., e21729. doi:10.1002/mas. 21729

Xiao, J. F., Zhou, B., and Ressom, H. W. (2012). Metabolite Identification and Quantitation in LC-MS/MS-based Metabolomics. Trac Trends Anal. Chem. 32, 1-14. doi:10.1016/j.trac.2011.08.009

Yang, Q.-J., Zhao, J.-R., Hao, J., Li, B., Huo, Y., Han, Y.-L., et al. (2018). Serum and Urine Metabolomics Study Reveals a Distinct Diagnostic Model for Cancer Cachexia. J. Cachexia, Sarcopenia Muscle 9 (1), 71-85. doi:10.1002/jcsm.12246

Yang, W., Huang, J., Wu, H., Wang, Y., Du, Z., Ling, Y., et al. (2020). Molecular Mechanisms of Cancer Cachexia-induced M-uscle A-trophy (Review). Mol. Med. Rep. 22 (6), 4967-4980. doi:10.3892/mmr.2020.11608

Yin, P., Lehmann, R., and Xu, G. (2015). Effects of Pre-analytical Processes on Blood Samples Used in Metabolomics Studies. Anal. Bioanal. Chem. 407 (17), 4879-4892. doi:10.1007/s00216-015-8565-x

Zhou, L., Zhang, T., Shao, W., Lu, R., Wang, L., Liu, H., et al. (2021). Amiloride Ameliorates Muscle Wasting in Cancer Cachexia through Inhibiting TumorDerived Exosome Release. Skeletal Muscle 11 (1), 17. doi:10.1186/s13395-02100274-5

Zhou, X., Wang, J. L., Lu, J., Song, Y., Kwak, K. S., Jiao, Q., et al. (2010). Reversal of Cancer Cachexia and Muscle Wasting by ActRIIB Antagonism Leads to Prolonged Survival. Cell 142 (4), 531-543. doi:10.1016/j.cell.2010.07.011

Conflict of Interest: The authors declare that the research was conducted in the absence of any commercial or financial relationships that could be construed as a potential conflict of interest.

Publisher's Note: All claims expressed in this article are solely those of the authors and do not necessarily represent those of their affiliated organizations, or those of the publisher, the editors, and the reviewers. Any product that may be evaluated in this article, or claim that may be made by its manufacturer, is not guaranteed or endorsed by the publisher.

Copyright $\odot 2022$ Cui, Li, Huang, Li and Lin. This is an open-access article distributed under the terms of the Creative Commons Attribution License (CC $B Y$ ). The use, distribution or reproduction in other forums is permitted, provided the original author(s) and the copyright owner(s) are credited and that the original publication in this journal is cited, in accordance with accepted academic practice. No use, distribution or reproduction is permitted which does not comply with these terms. 